\title{
Aortic Neocuspidization with Autologous Pericardium: Initial Experience of Single Center
}

\author{
Kubilay Karabacak ${ }^{*}$, Emre Kubat1, Gökhan Erol1, Murat Kadan1, Furkan Burak Akyol1, \\ Elgin Hacızade1, Suat Doganci', Mehmet Emin Ince'2, Uygar Cagdas Yuksel ${ }^{3}$, Murat Celik³, \\ Cengiz Bolcal ${ }^{1}$
}

\footnotetext{
${ }^{1}$ Department of Cardiovascular Surgery, Gülhane School of Medicine, Health Sciences University, Ankara, Turkey

${ }^{2}$ Department of Anesthesiology, Gülhane School of Medicine, Health Sciences University, Ankara, Turkey

${ }^{3}$ Department of Cardiology, Gülhane School of Medicine, Health Sciences University, Ankara, Turkey

Email: ^kubilaykarabacak@yahoo.com
}

How to cite this paper: Karabacak, K., Kubat, E., Erol, G., Kadan, M., Akyol, F.B., Hacizade, E., Doganci, S., Ince, M.E., Yuksel, U.C., Celik, M. and Bolcal, C. (2021) Aortic Neocuspidization with Autologous Pericardium: Initial Experience of Single Center. World Journal of Cardiovascular Surgery, 11, 51-60.

https://doi.org/10.4236/wjcs.2021.116008

Received: June 2, 2021

Accepted: June 27, 2021

Published: June, 30, 2021

Copyright $\odot 2021$ by author(s) and Scientific Research Publishing Inc. This work is licensed under the Creative Commons Attribution International License (CC BY 4.0).

http://creativecommons.org/licenses/by/4.0/

\begin{abstract}
Background: Aortic neo-cuspidization (AVNeo) procedure has been adopted by limited centers with the publication of the mid-long term successful results. The aim of this study was to present initial experience of the AVNeo procedure of a single center. Methods: The medical records of 24 patients who underwent AVNeo with or without concomitant cardiac surgery between February 2019 and February 2021 at our tertiary hospital were scanned retrospectively. Results: The mean age of patients was aged $58.21 \pm 13.14$ years and $16(66.7 \%)$ of them were men. 16 patients were operated on for aortic stenosis $(66.67 \%)$. Morphology of the aortic valve was tricuspid in $21(87.5 \%)$ and bicuspid in $3(12.5 \%)$ of the patients. Additional cardicac surgery was performed in 13 (54.17\%) patients. No patients needed reoperation for bleeding, pacemaker implantations, conversion to classical prosthetic aortic valve replacement, or infective endocarditis. Two patients died due to non-cardiac reasons. Preoperative peak and mean aortic valve pressures improved significantly at $1^{\text {st }}$ and $6^{\text {th }}$ months (Preop: $89.06 \pm 21.88 \mathrm{mmHg}$ and $56.38 \pm 15.09$ mmHg $1^{\text {st }}$ month: $22.00 \pm 3.93 \mathrm{mmHg}$ and $8.73 \pm 2.60 \mathrm{mmHg}$, $6^{\text {th }}$ month: 18.13 $\pm 3.02 \mathrm{mmHg}$ and $6.93 \pm 1.83 \mathrm{mmHg}$ ). Conclusion: In conclusion, the AVNeo procedure is a feasible technique for aortic valve pathologies, with the advantages of avoiding anticoagulants and the applicability of concomitant surgical procedures. Although this procedure requires meticulous experience, results similar to the available published literature can be obtained and reproducible even during learning curve when technical steps are strictly followed.
\end{abstract}

\section{Keywords}

Ozaki Procedure, Aortic Valve, Repair, Autologous Pericardium 


\section{Introduction}

Aortic valve replacement (AVR) with mechanical or bioprosthetic valves is still a standart approach for aortic valve diseases. Despite the durability, due to the need for long-term anticoagulation, morbidity and mortality such as bleeding and thromboembolic events reduce the desire of surgeons to use mechanical valves. For aortic valve disease, bioprosthetic valves are increasingly being used in elderly patients for replacement. However, despite advances in the design and construction of prosthetic valves, hemodynamic performance cannot yet be compared to that of natural aortic valves. Aortic valve repair is performed infrequently only by some experienced centers for only a selected group of aortic valve disease patients due to technical difficulties and durability of applied techniques. Thus, aortic valve neocuspidization (AVNeo) has emerged as another option that can be applied to a wide spectrum of aortic pathologies. Aortic valve reconstruction with a glutaraldehyde-treated autologous pericardium in patients with aortic valve disease was proposed and started by Duran et al. in 1995 [1]. Since then, different techniques have been reported. In 2011, Ozaki et al. published their first case series of aortic valve neocuspidisation for aortic valve disease with glutaraldehyde-treated autologous pericardium in 88 patients between April 2007 and August 2009 [2]. One of the main advantages of this method, the natural aortic root expansion in systole with maximal effective orifice area was achieved when compared with aortic valve replacement. AVNeo procedure has been adopted by limited centers with the publication of the mid-long term successful results. In our department, we started performing the Ozaki procedure for patients with aortic valve disease in February 2019. The aim of our study was to present short and mid-term results of the Ozaki procedure.

\section{Methods}

\subsection{Study Design}

24 patients who underwent AVNeo with or without concomitant cardiac surgery between February 2019 and February 2021 in the cardiac surgery department of our university hospital were included in this study. Patient's medical records were prospectively gathered and retrospectively analyzed from the electronic database of our hospital. The study protocol was approved by the local ethics committee (date: January 28, 2021; no. 2021/45). The study was conducted in accordance with the principles of the Declaration of Helsinki. In the current study, patients' preoperative clinical features, operative data (including aortic cusps sizes) and postoperative echocardiographic results were recorded.

\subsection{Surgical Technique}

This technique is routinely performed with median sternotomy incision with standardized aorto-caval/bicaval cannulation. Briefly, pericardial patches should be taken with care and treated with glutaraldehyde solutions. After resection of aortic cusps, the new pericardial aortic cusps are sewn into aortic annulus ac- 
cording to measurement of each aortic cusp. This sizing is crucial for procedural success. After sewing these three cusps, aortotomy incision was closed as usual, and the operation was completed with standardized techniques. Surgical steps of this technique for cuspis preparation and implantation have been explained in detail in previous articles [2] [3] [4] [5] [6].

\subsection{Statistics}

Statistical analysis was performed using the SPSS for Windows version 15.0 (SPSS Inc., Chicago, IL, USA). Continuous variables are expressed as mean \pm standard deviation (SD) and categorical variables as numbers (n) and percentages (\%). Friedman tests were conducted to test whether there is a significant change in the peak and mean aortic valve pressure, due to violations of parametric test assumptions (non-normal distribution and low number of cases). The Wilcoxon tests were performed to test the significance of pairwise differences using Bonferroni correction to adjust for multiple comparisons.

\section{Results}

\subsection{Preoperative Characteristics}

The mean age of patients was $58.21 \pm 13.14$ ( $\min -\max : 24-79)$ years and 16 (66.7\%) of them were male. The mean body surface area of the patients was 28.3 $\pm 3.7 \mathrm{~kg} / \mathrm{m}^{2}$. The distribution of the comorbidities was as follows: Coronary artery disease in $3(12.5 \%)$, hypertension in $5(20.8 \%)$, diabetes in $8(33.3 \%)$ and COPD in $4(16.7 \%)$ of the patients. Preoperative characteristics of each patient are listed in Table 1. Preoperative echocardiographic measurements of the patients revealed the mean left ventricular ejection fraction of $59 \% \pm 7.2 \%$ and mean aortic annulus diameter of $23.1 \pm 2.8 \mathrm{~mm} .13$ (54.17\%) patients needed concomitant cardiac procedures. 16 (66.67\%) patients presented with aortic stenosis (AS). Preoperative echocardiography showed an average peak and mean aortic valve pressures as $89.06 \pm 21.88 \mathrm{mmHg}$ and $56.38 \pm 15.09 \mathrm{mmHg}$, respectively. Morphology of the aortic valve was tricuspid in 21 (87.5\%) and bicuspid was in $3(12.5 \%)$ of the patients. The mean Society of Thoracic Surgeons score and logistic Euroscore were $1.16 \pm 1(0.33-3.92)$ and $4.41 \pm 4.92$ (0.46 - 17.05), respectively.

\subsection{Operative Data}

Patient's operative data and aortic cusps sizes were presented in Table 1. Concomitant procedures included 5 mitral valve replacements, 1 mitral valve repair, 3 coronary artery bypass surgeries, 3 ascending aortic replacements, 1 left atrial appendage internal plication and 1 pulmonary valve replacement operation. Aortic cups sizes were presented in Table 1. Mean aortic cross-clamping (ACC) time was $118.1 \pm 33.2$ ( $\min -\max : 67-173)$ minutes and total cardiopulmoner bypass (CPB) time was $162 \pm 45.7$ (min - max: 97 - 245) minutes. For isolated aortic valve pathologies, mean ACC time and CPB time were $93.3 \pm 18.2$ (min - max: 
Table 1. Patients baseline characteristics, operative data and postoperative results.

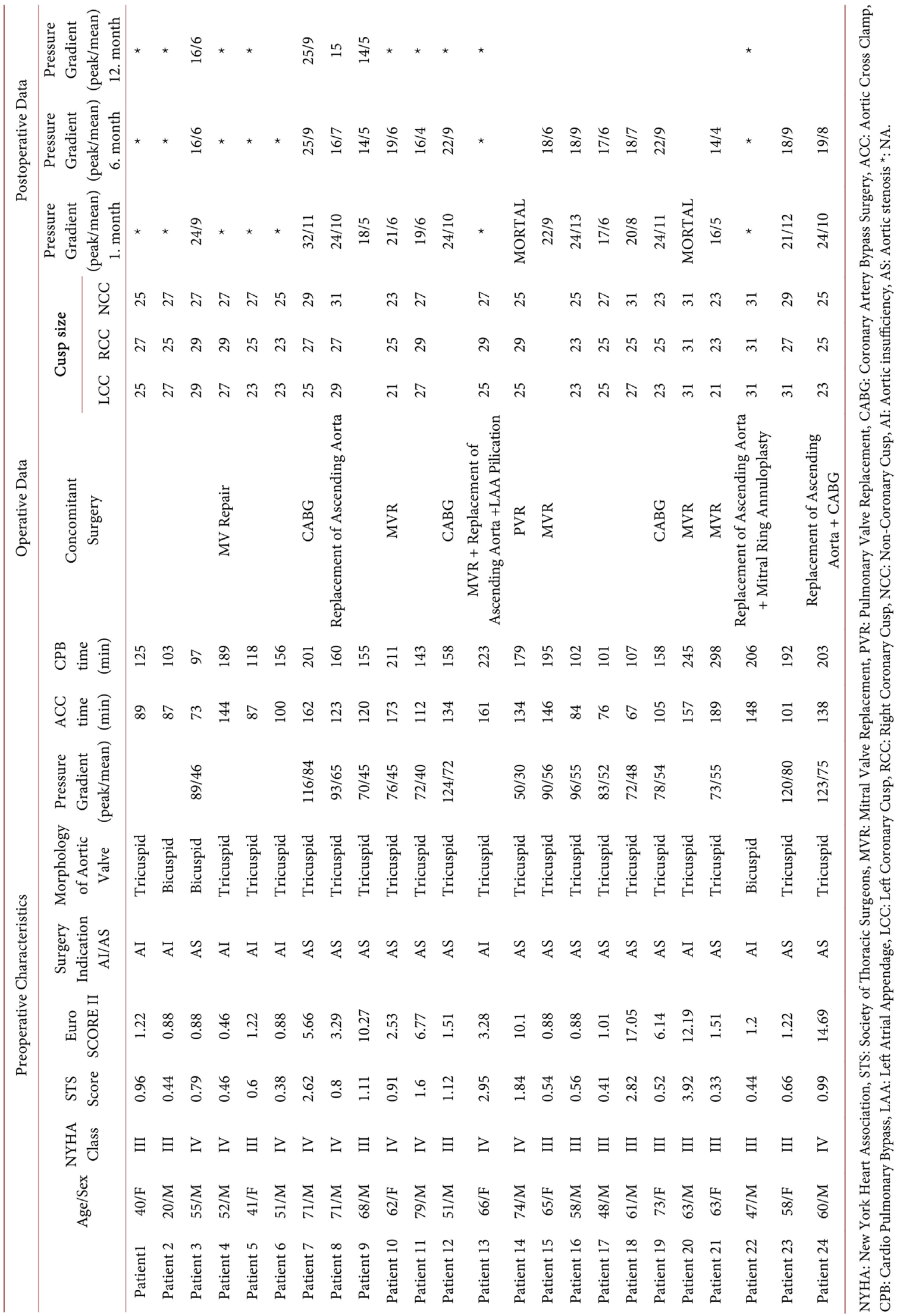


67 - 123) minutes and $130.3 \pm 31.6$ ( $\min -\max : 97$ - 197) minutes, respectively. There was no conversion to classical prosthetic AVR.

\subsection{Postoperative Data}

Patients' postoperative results were presented in Table 1 . The observed mean postoperative ventilation time in intensive care unit (ICU) was $6.5 \pm 5.3$ hours, mean ICU length of stay $1.6 \pm 1.1$ days, mean hospitalization length $9.7 \pm 7$ days. No patients needed reoperation for bleeding, pacemaker implantations or infective endocarditis. Postoperative atrial fibrilation occured in 1 patient who underwent concominant mitral valve replacement. During hospitalization, 2 (8.3\%) deaths from non-cardiac causes were recorded. Mesenteric ischemia was developed in 1 patient on the $3^{\text {rd }}$ postoperative day and 1 patient had ischemic cerebrovascular event on the $7^{\text {th }}$ postoperative day. The 30 -day mortality was also 2 $(8.3 \%)$ and included these aforementioned 2 patients. One of the patients with AS (patient 18) was reoperated in the 2 nd postoperative month due to grade 2 aortic insufficiency (AI).

In the patient group who were operated on for AI, worsening in the grade of aortic regurgitation was not observed. After surgery, trickle aortic regurgitation was seen in 2 of 8 patients. No or only trace aortic regurgitation was seen in rest of the patients.

At the first postoperative month, echocardiographic evaluation was performed in 15 of 16 patients with AS. Average peak and mean aortic valve pressure was $22.00 \pm 3.93 \mathrm{mmHg}$ and $8.73 \pm 2.60 \mathrm{mmHg}$ (both $\mathrm{p}=0.001$ ). At the $6^{\text {th }}$ postoperative month, echocardiographic evaluation was performed in all of these 15 patients. Average peak aortic valve pressure was $18.13 \pm 3.02 \mathrm{mmHg}$ (compared to both preoperative pressure and first month pressure, $\mathrm{p}=0.001$ ) and mean aortic valve pressure was $6.93 \pm 1.83 \mathrm{mmHg}$ (compared to preoperative pressure $\mathrm{p}=0.001$, and compared to first month pressure, $\mathrm{p}=0.002$ ).

For 2 patients who underwent concomitant mitral valve surgery, anticoagulant therapy with Warfarin sodium was employed at theuropatic dosages in the first 3 months and thereafter acetylsalicylic acid (100 mg/day) was given for life long. Patients who underwent isolated AVNeo procedure were treated with asetilsalicylic acid (100 mg/day) for 1 month after surgery and then it was deceased.

\section{Discussion}

The most common surgical treatment method for aortic valve pathologies is still aortic valve replacement. Despite all advances in prosthetic valve technologies, the ideal prosthetic valve still hasn't been developed. However, the implantation of the prosthetic valves to the aortic annulus brings along some anatomical and physiological issues due to the stent frame or the base ring of the valve. Prothesis-patient mismatch may occur as a result of impairment of the physiologic mobility of the aortic annulus and decrease of the aortic valve area. These problems may arise at similar rates in patients who underwent surgery as well as 
transcatheter aortic valve replacement (TAVR) [7] [8] [9]. Additionally, while bioprosthetic valves have a high risk of potential degeneration; mechanical prosthetic valves possess the risks of bleeding vs thromboembolic complication associated with anticoagulants.

AVNeo procedure has been performed since 2007 [2]. This procedure can be applied to all aortic valve pathologies. The biggest advantage of this technique is letting the anatomical and physiological movement of the aortic annulus and having an effective orifice area close to the native aortic valve. Glutaraldehyde-treated autologous pericard seems to provide mechanical and haemodynamic performance similar to the native aortic valve [10]. Since the 1960s, despite the description of many aortic valve repair techniques by using autologous or bovine pericardium, those methods haven't been widely adopted because of the calcification and the degeneration [11] [12] [13] [14]. Although using autologus pericardium has important advantages, fixation by glutaraldehyde to prevent retraction or thickening and to ensure the durability emerges problems about cytotoxicity [15]. Therefore, the search for a biocompatible material that will provide a lifelong solution to the patients is going on [16].

Ozaki procedure can be applied to every kind of aortic valve diseases such as AS, AI, or combined aortic valve pathologies. It can also successfully be used for congenital abnormalities such as bicuspid and unicuspid aorta [6] [17]. In this patient cohort, 3 (2 with AI, 1 with AS) (12.5\%) patients were operated on due to bicuspid aorta and their postoperative results were similar to tricuspid patients.

Ozaki procedure is accepted to be a safe technique for young patients as well. In this series, 12 (50\%) patients were under 60-year-old. No mortality and reoperation were noted in our younger patients and all of them showed uneventful follow-up. Similarly, in a study conducted in patients younger than 60 years old, the freedom from reoperetion rate was found $98.9 \%$ at 76 months follow up [18]. While these are not long-term consequences, available results can be accepted as promising for the young patient population in the long term.

According to the surgical data of our patients mean ACC time and CPB time were $118.1 \pm 33.2(\min -\max : 67-173)$ minutes and $162 \pm 45.7$ (min - $\max : 97$ 245) minutes respectively. Although these durations were longer than the durations of Arutyunyan et al., in their study the rate of patients who underwent isolated AVNeo was higher than the rate of patients in our study [19]. In our study, in patients who underwent isolated AVNeo procedure, mean ACC time and CPB time $93.3 \pm 18.2$ were $130.3 \pm 31.6$ minutes respectively. In consistent with our results, Ozaki et al. reported that the ACC time was $110.1 \pm 26.8$ minutes in isolated aortic valve procedures [3]. Also, ACC and CPB times may even be decreased with the completion of learning curve. In the study from Ozaki et al., mean ACC time was decreased to as $96.7 \pm 26.9$ minutes for the patients who were operated on in the last 10 months.

In our study, 16 patients with AS were undergone AVNeo with or without concomitant surgery and both statistically and clinically improvement in aortic valve pressure was obtained after surgery. Only 1 patient, who was applied an 
isolated AVNeo procedure for AS, was reoperated due to central AI on the $2^{\text {nd }}$ postoperative month. On the $2^{\text {nd }}$ postoperative month, the echocardiographic examination was performed due to his complaints of dyspnea and grade 2 central AI with hypertrophic ventricle was detected. Due to unresponsiveness to medical treatment, sutureless AVR was performed. This patient had a much wider non-coronary leaflet than the other leaflets and the rudimentary commissure between non-coronary and left coronary leaflets was located lower than the other commissural level. In addition to the coaptation defect of glutaraldehyde-treated leaflets, these differences in the commissural levels contributed to the development central AI. Very recently, Ozaki recommended creating a new commissure if the difference between measurements of each cusp is greater than $2 \mathrm{~mm}[20]$.

In another study, Iida et al. performed AVNeo in 57 patients with AS [21]. Similarly, to our result, they reported that preoperative average peak pressure gradient was reduced from $89 \pm 32.9 \mathrm{mmHg}$ to $22 \pm 10.7 \mathrm{mmHg}$ at the $1^{\text {st }}$ postoperative week and to $19.2 \pm 9.7 \mathrm{mmHg}$ at postoperative $20^{\text {th }}$ month. Only 2 patients were reoperated due to infective endocarditis and recurrence of aortic regurgitation. In another study conducted on 77 patients who mostly had AS, Krane et al., reported that moderate-severe AI was developed in 2 patients. In the same study, they denoted that the hospitalization times were similar to those of prosthetic AVR patients [22]. Also, studies that compared AVNeo with the aortic valve replacement reported that greater effective aortic valve area and smaller aortic valve pressure gradient pressure were obtained with AVNeo procedure [23] [24]. In addition, aortic annular dimensions after AVNeo were found similar to the dimensions of normal aortic valves [23].

In addition, the results of several studies with more patients and longer follow-up were showed that the drop in aortic pressure gradient after AVNeo operation was maintained for a long time and cumulative incidence of reoperation was too low. Ozaki et al. carried out a retrospective study which included 404 patients with $23.7 \pm 13.1$ months of the mean follow-up period. The mean preoperative average peak pressure gradient of patients with AS decreased from 79.6 $\pm 32.5 \mathrm{mmHg}$ to $19.8 \pm 10.2 \mathrm{mmHg}$ at the first postoperative week and to $13.8 \pm$ $3.7 \mathrm{~mm} \mathrm{Hg} 3.5$ years after surgery. Conversion to prosthetic valve replacement and thromboembolic events were not recorded. Freedom from reoperation was reported as $96.2 \%$ at 53 months of follow-up [3]. In another study by Ozaki et al., which included 850 patients with various aortic valve diseases with a mean follow-up of $53.7 \pm 28.2$ months, actuarial freedom from death, the cumulative incidence of reoperation and recurrence of moderate or severe aortic regurgitation were found as $85.9 \%, 4.2 \%$ and $7.3 \%$, respectively. The preoperative echocardiography revealed the mean diameter of the aortic annulus of $20.9 \pm 3.3 \mathrm{~mm}$ for all patients and the mean peak pressure gradient of $68.9 \pm 36.3 \mathrm{~mm} \mathrm{Hg}$ for the patient with AS. The mean peak pressure gradient was reduced to $19.5 \pm 10.3$ $\mathrm{mmHg}$ at the 1st postoperative week and to $5.2 \pm 6.3 \mathrm{mmHg}$ at the 8 th postoperative years [6]. 
Our study was conducted on a single center and with a small number of patients. The lack of long-term follow-up is the major limitation of this study. Even though the performed procedure has more favorable mid-term results, the long-term durability of the glutaraldehyde-treated pericardium does not exist yet on the patients who underwent AVNeo procedure.

In conclusion, AVNeo is a feasible and reproducible procedure with successful early postoperative hemodynamic results and with the advantages of no anticoagulants necessity, and it can be performed concomitantly with other cardiac surgical interventions. In summary, our results are similar to Ozaki's own series results. Although this procedure requires meticulous experience, results similar to the available published literature can be obtained and reproducible even during the learning curve when technical steps are strictly followed. Therefore, this procedure can be considered equivalent to a standard valve surgical procedure.

\section{Conflicts of Interest}

The authors declare no conflict of interest.

\section{References}

[1] Duran, C.M., Gometza, B., Kumar, N., Gallo, R. and Martin-Duran, R. (1995) Aortic Valve Replacement with Freehand Autologous Pericardium. The Journal of Thoracic and Cardiovascular Surgery, 110, 511-516. https://doi.org/10.1016/S0022-5223(95)70248-2

[2] Ozaki, S., Kawase, I., Yamashita, H., Uchida, S., Nozawa, Y., Matsuyama, T., et al. (2011) Aortic Valve Reconstruction Using Self-Developed Aortic Valve Plasty System In Aortic Valve Disease. Interactive Cardiovascular and Thoracic Surgery, 12 550-553. https://doi.org/10.1510/icvts.2010.253682

[3] Ozaki, S., Kawase, I., Yamashita, H., Uchida, S., Nozawa, Y., Takatoh, M., et al. (2014) A Total of 404 Cases of Aortic Valve Reconstruction with Glutaraldehyde-Treated Autologous Pericardium. The Journal of Thoracic and Cardiovascular Surgery, 147, 301-306. https://doi.org/10.1016/j.jtcvs.2012.11.012

[4] Ozaki, S., Kawase, I., Yamashita, H., Uchida, S., Nozawa, Y., Takatoh, M., et al. (2014) Aortic Valve Reconstruction Using Autologous Pericardium for Ages over 80 Years. Asian Cardiovascular and Thoracic Annals, 22, 903-908. https://doi.org/10.1177/0218492314520748

[5] Ozaki, S., Kawase, I., Yamashita, H., Uchida, S., Takatoh, M., Hagiwara, S., et al. (2015) Aortic Valve Reconstruction Using Autologous Pericardium for Aortic Stenosis. Circulation Journal, 79, 1504-1510. https://doi.org/10.1253/circj.CJ-14-1092

[6] Ozaki, S., Kawase, I., Yamashita, H., Uchida, S., Takatoh, M. and Kiyohara, N. (2018) Midterm Outcomes after Aortic Valve Neocuspidization with Glutaraldehyde-Treated Autologous Pericardium. The Journal of Thoracic and Cardiovascular Surgery, 155, 2379-2387. https://doi.org/10.1016/j.jtcvs.2018.01.087

[7] Mooney, J., Sellers, S. L., Blanke, P., Pibarot, P., Hahn, R. T., Dvir, D., et al. (2017) CT-Defined Prosthesis-Patient Mismatch Downgrades Frequency and Severity, and Demonstrates No Association with Adverse Outcomes after Transcatheter Aortic Valve Replacement. JACC: Cardiovascular Interventions, 10, 1578-1587. https://doi.org/10.1016/j.jcin.2017.05.031 
[8] Zorn, G.L., Little, S.H., Tadros, P., Deeb, G.M., Gleason, T.G., Heiser, J., et al. (2016) Prosthesis-Patient Mismatch in High-Risk Patients with Severe Aortic Stenosis: A Randomized Trial of a Self-Expanding Prosthesis. The Journal of Thoracic and Cardiovascular Surgery, 151, 1014-1023. https://doi.org/10.1016/j.jtcvs.2015.10.070

[9] Ghanta, R.K. and Kron, I.L. (2016) Patient-Prosthesis Mismatch: Surgical Aortic Valve Replacement Versus Transcatheter Aortic Valve Replacement in High Risk Patients with Aortic Stenosis. Journal of Thoracic Disease, 8, E1441-E1443. https://doi.org/10.21037/jtd.2016.09.62

[10] Meuris, B., Ozaki, S., Neethling, W., De Vleeschauwer, S., Verbeken, E., Rhodes, D., et al. (2016) Trileaflet Aortic Valve Reconstruction with a Decellularized Pericardial Patch in a Sheep Model. The Journal of Thoracic and Cardiovascular Surgery, 152, 1167-1174. https://doi.org/10.1016/j.jtcvs.2016.05.024

[11] Bjoerk, V.O. and Hultquist, G. (1964) Teflon and Pericardial Aortic Valve Prostheses. The Journal of Thoracic and Cardiovascular Surgery, 47, 693-701. https://doi.org/10.1016/S0022-5223(19)33497-X

[12] Bahnson, H.T., Hardesty, R.L., Baker, L.D., Jr., Brooks, D.H. and Gall, D.A. (1970) Fabrication and Evaluation of Tissue Leaflets for Aortic and Mitral Valve Replacement. Annals of Surgery, 171, 939-947. https://doi.org/10.1097/00000658-197006010-00015

[13] Ross, D.N. (1963) Surgical Reconstruction of the Aortic Valve. Lancet, 281, 571-574. https://doi.org/10.1016/S0140-6736(63)92687-4

[14] Senning A. (1967) Fascia Lata Replacement of Aortic Valves. The Journal of Thoracic and Cardiovascular Surgery, 54, 465-470. https://doi.org/10.1016/S0022-5223(19)43054-7

[15] Kumar, S.P., Prabhakar, G., Kumar, M., Kumar, N., Shahid, M., Ali, M.L., et al. (1995) Comparison of Fresh and Glutaraldehyde-Treated Autologous Stented Pericardium as Pulmonary Valve Replacement. Journal of Cardiac Surgery, 10, 545-551. https://doi.org/10.1111/j.1540-8191.1995.tb00630.x

[16] Jayakrishnan, A. and Jameela, S.R. (1996) Glutaraldehyde as a Fixative in Bioprostheses and Drug Delivery Matrices. Biomaterials, 17, 471-484.

https://doi.org/10.1016/0142-9612(96)82721-9

[17] Kawase, I., Ozaki, S., Yamashita, H., Uchida, S., Nozawa, Y., Matsuyama, T., et al. (2012) Aortic Valve Reconstruction of Unicuspid Aortic Valve by Tricuspidization Using Autologous Pericardium. The Annals of Thoracic Surgery, 94, 1180-1184. https://doi.org/10.1016/j.athoracsur.2012.05.016

[18] Ozaki, S., Kawase, I., Yamashita, H., Nozawa, Y., Takatoh, M., Hagiwara, S. et al. (2014) Aortic Valve Reconstruction Using Autologous Pericardium for Patients Aged Less Than 60 Years. The Journal of Thoracic and Cardiovascular Surgery, 148, 934-938. https://doi.org/10.1016/j.jtcvs.2014.05.041

[19] Arutyunyan, V., Chernov, I., Komarov, R., Sinelnikov, Y., Kadyraliev, B., Enginoev, S., et al. (2020) Immediate Outcomes of Aortic Valve Neocuspidization with Glutaraldehyde-Treated Autologous Pericardium: A Multicenter Study. Brazilian Journal of Cardiovascular Surgery, 35, 241-248. https://doi.org/10.21470/1678-9741-2020-0019

[20] Ozaki S. (2019) Ozaki Procedure: 1,100 Patients with Up to 12 Years of Follow-Up. Turk Gogus Kalp Damar Cerrahisi Dergisi, 27, 454 p. https://doi.org/10.5606/tgkdc.dergisi.2019.01904

[21] Iida, Y., Fujii, S., Akiyama, S. and Sawa, S. (2018) Early and Mid-Term Results of 
Isolated Aortic Valve Neocuspidization in Patients with Aortic Stenosis. General Thoracic and Cardiovascular Surgery, 66, 648-652.

https://doi.org/10.1007/s11748-018-0976-0

[22] Krane, M., Boehm, J., Prinzing, A., Ziegelmueller, J., Holfeld, J. and Lange, R. (2021) Excellent Hemodynamic Performance after Aortic Valve Neocuspidization Using Autologous Pericardium. The Annals of Thoracic Surgery, 111, 126-133.

https://doi.org/10.1016/j.athoracsur.2020.04.108

[23] Yamamoto, Y., Iino, K., Shintani, Y., Kato, H., Kimura, K., Watanabe, G., et al. (2017) Comparison of Aortic Annulus Dimension after Aortic Valve Neocuspidization with Valve Replacement and Normal Valve. Seminars in Thoracic and Cardiovascular Surgery, 29, 143-149. https://doi.org/10.1053/j.semtcvs.2016.11.002

[24] Wiggins, L.M., Mimic, B., Issitt, R., Ilic, S., Bonello, B., Marek, J., et al. (2020) The Utility of Aortic Valve Leaflet Reconstruction Techniques in Children and Young Adults. The Journal of Thoracic and Cardiovascular Surgery, 159, 2369-2378.

https://doi.org/10.1016/j.jtcvs.2019.09.176 